\title{
BMJ Open Quality Appropriate utilisation of cardiac telemetry monitoring: a quality improvement project
}

To cite: Stoltzfus KB, Bhakta M, Shankweiler C, et al. Appropriate utilisation of cardiac telemetry monitoring: a quality improvement project. BMJ Open Quality 2019;8:e000560. doi:10.1136/ bmjoq-2018-000560

Received 22 October 2018 Revised 19 December 2018 Accepted 23 March 2019
Check for updates

(C) Author(s) (or their employer(s)) 2019. Re-use permitted under CC BY-NC. No commercial re-use. See rights and permissions. Published by BMJ.

${ }^{1}$ Department of Internal Medicine and Palliative Medicine, The University of Kansas Health System, Kansas City, Kansas, USA

${ }^{2}$ Department of Internal Medicine, The University of Kansas Health System, Kansas City, Kansas, USA

Correspondence to

Dr Ky B Stoltzfus;

kstoltzfus@kumc.edu

\section{Ky B Stoltzfus, ${ }^{1}$ Maharshi Bhakta, ${ }^{2}$ Caylin Shankweiler, ${ }^{2}$ Rebecca R Mount, ${ }^{2}$ Cheryl Gibson ${ }^{2}$}

\section{ABSTRACT}

For hospitals located in the United States, appropriate use of cardiac telemetry monitoring can be achieved resulting in cost savings to healthcare systems. Our institution has a limited number of telemetry beds, increasing the need for appropriate use of telemetry monitoring to minimise delays in patient care, reduce alarm fatigue, and decrease interruptions in patient care.

This quality improvement project was conducted in a single academic medical centre in Kansas City, Kansas. The aim of the project was to reduce inappropriate cardiac telemetry monitoring on intermediate care units. Using the 2004 American Heart Association guidelines to guide appropriate telemetry utilisation, this project team sought to investigate the effects of two distinct interventions to reduce inappropriate telemetry monitoring huddle intervention and mandatory order entry. Telemetry utilisation was followed prospectively for 2 years.

During our initial intervention, we achieved a sharp decline in the number of patients on telemetry monitoring. However, over time the efficacy of the huddle intervention subsided, resulting in a need for a more sustained approach. By requiring physicians to input indication for telemetry monitoring, the second intervention increased adherence to practice guidelines and sustained reductions in inappropriate telemetry use.

\section{PROBLEM}

Cardiac telemetry monitoring plays an important role during acute hospitalisation for cardiac rate/rhythm monitoring, diagnosis of arrhythmias and myocardial ischaemia. ${ }^{1} \mathrm{It}$ requires resources of device infrastructure, device maintenance and human resources for oversight, which contribute to increased healthcare costs. ${ }^{2}$ In 2017, the American Heart Association (AHA) published updated practice standards for telemetry monitoring that addressed overuse, appropriate use, alarm management and documentation in electronic medical records (EMR). ${ }^{3}$ Effective use of telemetry monitoring with physician oversight, in the form of daily or weekly huddles, has shown to significantly reduce cost in patient care. ${ }^{45}$ Clinicians may order telemetry monitoring with the goal of closer monitoring a patient's vitals and clinical status. However, multiple studies have noted that overuse of telemetry monitoring rarely detects clinically significant events, ${ }^{6-8}$ causes alert fatigue ${ }^{9} 10$ and incurs unnecessary healthcare costs. ${ }^{11}$

In our institution, the availability of telemetry is one of the limiting factors for transfer of a patient from the emergency department or clinic space to an inpatient bed. Furthermore, the lack of available telemetry beds has a negative impact on throughput within the hospital. Our hospital admission order set requires physicians to select inpatient telemetry versus general medical/surgical status. However, there is no mechanism in place for discontinuation of telemetry once it is ordered for an admission. This has created prolonged unnecessary telemetry monitoring.

The project team began to examine the average rate of telemetry use in progressive care units across the institution, with the exclusion of labour and delivery, paediatric and intensive care units. We utilised the Plan, Do, Study, Act (PDSA) model for this quality improvement project. We defined telemetry rate as the number of patients with telemetry monitoring divided by the total number of patients on the observed units. The cumulative percentage of the hospital units was calculated, and baseline data reflected that $62.5 \%$ of patients on progressive care floors were on telemetry at any given time. Thus, we aimed to reduce the inappropriate utilisation of cardiac telemetry monitoring by $20 \%$, similar to the percentage reported by Chen et al, ${ }^{12}$ over a 2-year period using a quality improvement framework with two PDSA cycles.

\section{BACKGROUND}

Healthcare reform in the United States has heightened awareness for the need to improve quality of care while lowering costs. Reform efforts have pushed healthcare organisations to analyse and identify high cost practices and to address overuse and reduce waste 
to improve efficiency. ${ }^{6}$ Cardiac telemetry monitoring has been a focus area for overuse reduction since it expanded from monitoring high-risk patients with cardiac disease to inappropriately monitoring low-risk patients without cardiac disease. ${ }^{613}$ The Society of Hospital Medicine has specifically highlighted this problem in their choosing wisely recommendations. ${ }^{14}$

Considering the patient's level of severity, progressive care units were developed in efforts to accommodate those who needed more extensive care than general medicine but less than intensive care, allowing for additional telemetry monitoring in hospital settings. ${ }^{15}$ The original guidelines for inpatient telemetry (ECG) monitoring were released by the AHA in $2004^{16}$ and the guidelines were updated in 2017 to address appropriate use of ECG monitoring. ${ }^{3}$ The updated practice guidelines provide class I, II and III indications for the use of telemetry, from when telemetry monitoring should be performed to when monitoring is not recommended. Subsequent to the publication of the 2004 practice guidelines, significant concerns regarding overuse and inappropriate use of telemetry monitoring in the progressive care setting developed.

Findings from various published studies indicate telemetry monitoring is often overused in intermediate level of care settings. ${ }^{6} 1113$ Benjamin et al analysed utilisation patterns of cardiac telemetry use on four academic telemedicine floors and discovered that $33 \%$ of telemetry days did not meet appropriate indications for telemetry monitoring. ${ }^{6}$ To improve appropriate monitoring, the investigators suggested implementing quality improvement projects such as computerised order entry and decision tools to reduce waste. Dressler et al developed a telemetry order entry set that supported AHA guidelines to standardise the telemetry ordering process, which reduced telemetry use by $70 \%$ without hindering patient care. ${ }^{11}$ They estimated the reduction in telemetry use saved an estimated 4.8 million dollars annually, and lowered the mean duration of telemetry days by $43 \%$.

\section{MEASUREMENT}

Our hospital has an inpatient oversight committee, the Acute Care Committee (ACC), which meets on a monthly basis to address important patient care initiatives to improve quality, patient satisfaction, cost and throughput of the clinical operation. Inappropriate utilisation of cardiac telemetry monitoring was identified as a key area for improvement in all of these domains. Stakeholders, including attending physicians, resident physicians, bedside nurses and nurse managers, convened to understand the current state of telemetry utilisation at that time and develop interventions to reduce inappropriate telemetry utilisation. We developed two targeted interventions that were deployed sequentially and with embedded PDSA cycles within each intervention.

The percentage of patients on telemetry monitoring was extracted from hospital billing data. If a patient had an order for telemetry monitoring, then the order was submitted as a charge at midnight each calendar day. When the telemetry monitoring order was discontinued, then the charge would no longer be placed for the following calendar day.

\section{DESIGN}

The telemetry project was a 2-year intervention with two PDSA cycles. We collected pre-data and post-data for telemetry utilisation with each cycle. Data were collected quarterly and reported by eight hospital units that offer cardiac telemetry monitoring, including cardiothoracic progressive care, cardiovascular progressive care, medical telemetry/pulmonary hypertension progressive care, inpatient solid organ transplant/renal care, cardiac and family medicine progressive care, neuroscience progressive care, and two medical telemetry progressive care units. Intensive care, labour and delivery, and paediatric units were excluded from this project.

\section{STRATEGY}

Pilot

Before proceeding with PDSA cycle 1, we performed a pilot test on hospital unit B, which demonstrated feasibility for carrying out the intervention during the unit huddle. The pilot was conducted during a 30-day period on a single hospital unit (unit B) during Quarter 1, 2015. It allowed us to verify that physicians and nurses were able to communicate about reasons for telemetry use for individual patients, which would be important for the intervention in PDSA cycle 1. At the daily huddle, the bedside nurse for a patient asked the resident or attending physician, 'Does this patient still need telemetry monitoring?' If the physician replied 'yes', then the nurse would ask, 'What is the reason for continued telemetry monitoring?' The physician would provide the reason and the nurse would record the answer. If the physician replied 'no', then the nurse would ask the physician, 'Would you like me to place a verbal order to discontinue telemetry monitoring or will you do that?' The physician then stated the plan for discontinuation of telemetry monitoring for that patient. During this pilot phase, there was a slight reduction in telemetry utilisation for unit B from Quarter 2 to Quarter 3, 2014 (43.4\% vs 39.3\%).

\section{PDSA cycle 1: huddle intervention}

Our hospital has medical teams assigned geographically by hospital unit. For example, an Internal Medicine service will be primarily assigned to a particular unit and the vast majority of that team's patients will be located on that unit. The same is true for surgical and other specialty services. Each unit has a daily huddle where nurses, physicians, social worker, and a nurse case manager discuss ongoing treatment and discharge plans for each patient. There are a series of questions or discussion points shared about each patient during the course of the huddle. 
Once the pilot was determined to be feasible, the intervention was then taken hospital wide to all hospital units during Quarter 2, 2015 and continued until Quarter 3, 2015. The intervention was not specifically continued after Quarter 3, 2015 but may have had some residual effect if individual nurses continued the intervention thereafter. At the daily huddles on every unit, the bedside nurse asked the attending or resident physician, 'Does this patient still need telemetry monitoring?' A similar process of discontinuing telemetry was completed as described during the Pilot phase. The results for telemetry monitoring were tracked using run charts for each hospital unit and were presented on a quarterly basis to the ACC. This completed the first PDSA cycle for the project.

\section{PDSA cycle 2: admission telemetry monitoring order}

The first PDSA cycle did not decrease hospital-wide telemetry utilisation to the intended goal. Hence, the second intervention was implemented on a hospital-wide basis in Quarter 2, 2016 and remains in place. We implemented a hard-stop in the admission orders that forced the admitting physician to indicate a reason for telemetry monitoring from a list of pre-determined indications taken from the 2004 AHA guidelines available at that time. ${ }^{16}$ A hard-stop means that the physician could not proceed with submitting admission orders until this question was answered in the orders. We implemented a hard-stop due to lack of efficacy with a user-dependent change utilised in PDSA cycle 1. The hard-stop was counter balanced with a choice for 'other', wherein the provider could effectively bypass the decision point by entering his/ her own rationale, which allowed for fewer unintended consequences cited in prior studies. ${ }^{17}$ The reasons from which a physician could choose are indicated in table 1 . When the indication of 'other' was chosen, the admitting physician was required to enter a free-text response in an adjacent field. The 'other' responses were tabulated separately and contained written comments such as cardiac, neuro, non-cardiac surgery, electrolytes, anaemia, medication or drug, infection, pulmonary, and oncology. Indications were tabulated during the periods of Quarter 4, 2016 to Quarter 1, 2017.

We undertook a qualitative process of content analysing the open-ended 'other' responses by first having a research assistant hand tabulate the entries and group them into clinical categories. Two physicians then took these categories and further clustered them into broader clinical categories of indications, including neurological, cardiac, non-cardiac surgery, infectious, haematologic, electrolyte-related, medications or intoxications and no reason indicated. Subsequently, the same two physicians reviewed the list of 'other' indications together and made a judgement whether the indication was indicated, questionable, or not indicated based on 2004 AHA guidelines. The category of questionable reflects free-text responses where both physicians agreed that the reason listed may be indicated in the proper clinical context; however, a
Table 1 Hard-stop intervention reasons for telemetry

Reason
Pre-cardiac/post-cardiac disease
intervention

Cardiac catheterisation, coronary 1911 (17.7\%)

artery bypass surgery, cardiac

ablation, etc

\begin{tabular}{|c|c|}
\hline \multicolumn{2}{|l|}{ Supraventricular tachycardia } \\
\hline $\begin{array}{l}\text { Heart rate }>120 \text {, includes atrial } \\
\text { fibrillation or atrial flutter }\end{array}$ & $888(8.2 \%)$ \\
\hline \multicolumn{2}{|l|}{ Chest pain } \\
\hline Rule out myocardial infarction & $853(7.9 \%)$ \\
\hline $\begin{array}{l}\text { Acute heart failure on parenteral } \\
\text { therapy }\end{array}$ & $608(5.6 \%)$ \\
\hline \multicolumn{2}{|l|}{ Acute coronary syndrome } \\
\hline STEMI, NSTEMI, unstable angina & $569(5.3 \%)$ \\
\hline Syncope & $518(4.8 \%)$ \\
\hline Stroke work-up & $456(4.2 \%)$ \\
\hline \multicolumn{2}{|l|}{ Prolonged QTC monitoring } \\
\hline $\begin{array}{l}\text { Medication or intoxication } \\
\text { requiring cardiac monitoring }\end{array}$ & $402(3.7 \%)$ \\
\hline $\begin{array}{l}\text { Acute electrolyte abnormality with } \\
\text { ECG change }\end{array}$ & $277(2.6 \%)$ \\
\hline Pulmonary embolism & $139(1.3 \%)$ \\
\hline \multicolumn{2}{|l|}{$\begin{array}{l}\text { Non-sustained ventricular } \\
\text { tachycardia }\end{array}$} \\
\hline$>3$ consecutive beats and $<30 s$ & $116(1.1 \%)$ \\
\hline $\begin{array}{l}\text { Acute pericarditis/myocarditis/ } \\
\text { endocarditis }\end{array}$ & $28(0.3 \%)$ \\
\hline Post-cardiac arrest & $23(0.2 \%)$ \\
\hline Other & $3991(37.0 \%)$ \\
\hline Total & $10779(100 \%)$ \\
\hline
\end{tabular}

NSTEMI, non-ST-segment elevation myocardial infarction; QTC, corrected QT interval, as measured by electrocardiogram ; STEMI, ST-segment elevation myocardial infarction.

determination could not be made without an exhaustive chart review for each patient.

\section{RESULTS}

The hospital-wide intervention at huddles showed variable impact across different hospital units, as shown in table 2. Two units had a reduction in use as a result of the huddle intervention while the remaining had no sizeable change or increased during the intervention. The mean utilisation of telemetry across all hospital units had a small drop after the hospital-wide huddle intervention but then returned to previous baseline level within two subsequent quarters (see figure 1).

Initiation of the admission order intervention showed large reductions in mean telemetry utilisation consistently across all hospital units from a baseline prior to Quarter 2 of 2016 of $62.4 \%$ to a new baseline of $51.3 \%$ afterwards. 
Table 2 Telemetry utilisation rate by unit (\% patients with telemetry)

\begin{tabular}{|c|c|c|c|c|c|c|c|c|}
\hline \multirow[b]{2}{*}{ Unit } & \multicolumn{2}{|c|}{ Baseline } & \multicolumn{4}{|c|}{ Huddle intervention } & \multicolumn{2}{|c|}{ Admission order intervention } \\
\hline & $\begin{array}{l}2014 \\
\text { Q4 }\end{array}$ & $\begin{array}{l}2015 \\
\text { Q2 }\end{array}$ & $\begin{array}{l}2015 \\
\text { Q2 }\end{array}$ & $\begin{array}{l}2015 \\
\text { Q3 }\end{array}$ & $\begin{array}{l}2015 \\
\text { Q4 }\end{array}$ & $\begin{array}{l}2016 \\
\text { Q1 }\end{array}$ & $\begin{array}{l}2016 \\
\text { Q2 }\end{array}$ & $\begin{array}{l}2016 \\
\text { Q3 }\end{array}$ \\
\hline $\begin{array}{l}\text { Unit B } \\
\text { (Medical Telemetry Progressive Care) }\end{array}$ & $51 \%$ & $51 \%$ & $43 \%$ & $39 \%$ & $48 \%$ & $41 \%$ & $37 \%$ & $30 \%$ \\
\hline $\begin{array}{l}\text { Unit D } \\
\text { (Inpatient Solid Organ Transplant) }\end{array}$ & $59 \%$ & $66 \%$ & $55 \%$ & $45 \%$ & $60 \%$ & $54 \%$ & $54 \%$ & $40 \%$ \\
\hline $\begin{array}{l}\text { Unit E } \\
\text { (Pulmonary Hypertension Progressive Care) }\end{array}$ & $58 \%$ & $64 \%$ & $60 \%$ & $56 \%$ & $59 \%$ & $65 \%$ & $61 \%$ & $54 \%$ \\
\hline $\begin{array}{l}\text { Unit H } \\
\text { (Cardiovascular Progressive Care) }\end{array}$ & $77 \%$ & $79 \%$ & $80 \%$ & $71 \%$ & $78 \%$ & $79 \%$ & $77 \%$ & $69 \%$ \\
\hline
\end{tabular}

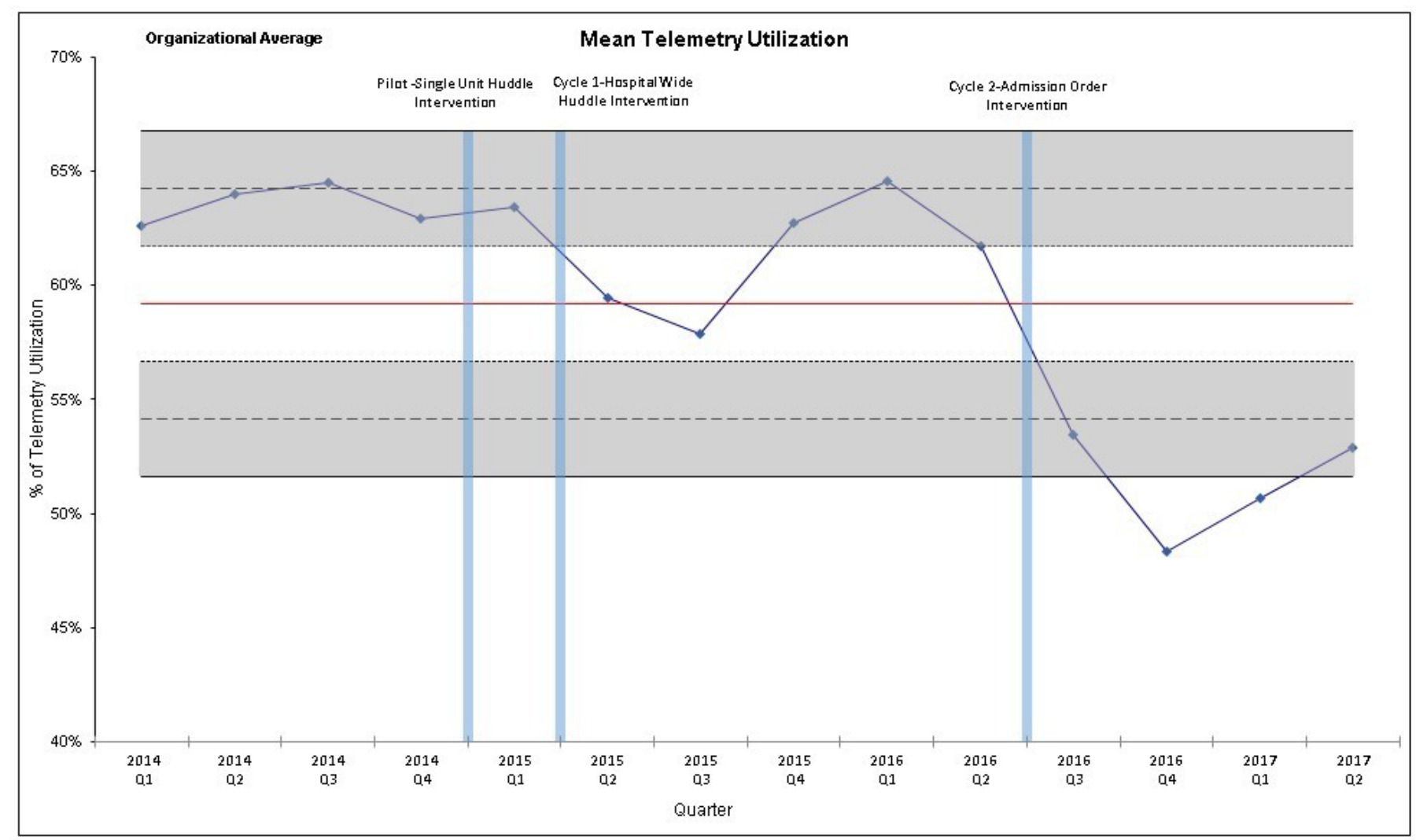

\footnotetext{
— Three Sigma Limit

--- Two Sigma Limit

-..-- One Sigma Limit Average
}

Figure 1 Telemetry utilisation statistical process control chart (SPC chart by proportion) for the entire hospital. 
This represents a $11.1 \%$ absolute reduction, or a $17.8 \%$ relative reduction, of telemetry utilisation across all hospital units. This reduction trend remained consistent after the admission order intervention (see figure 1).

The 'other' category accounted for a large number of entries after the admission order intervention was initiated, accounting for $37 \%$ of entries. Pre-cardiac/post-cardiac intervention accounted for the next most commonly chosen indication with $18 \%$ of entries. Additional indications chosen are shown in table 1.

The 'other' category is further subdivided into clinical categories with clinical judgement about whether the reason written in free text was indicated, not indicated or questionable. When the admitting physician selected the 'other' category, a third $(33.5 \%)$ of the responses were either blank or did not contain a reason for telemetry. When a reason was given, $19.1 \%$ of reasons were indicated according to AHA guidelines, $20.7 \%$ were questionable and $60.1 \%$ were not indicated.

\section{LESSONS AND LIMITATIONS}

Our study has a number of limitations. First, PDSA cycle 1 was essentially an intervention of education and gentle exhortation, which was not sustained. As discussed by Gosbee $e t a l$ in which they posited the concept of an effectiveness hierarchy of interventions, this type of intervention consistently achieves a smaller impact and typically does not have lasting effects. ${ }^{18} 19$ Second, PDSA cycle 2 involved a hard-stop in admission orders and led to a greater decrease in telemetry utilisation. However, for more than a third of entries ordering providers used the 'other' category, often specifying legitimate indications that were already listed on the menu of choices. Many entries were inappropriate or left blank, which we perceive as the ordering provider circumventing the intervention.

At the initiation of the project, we did not include specific balancing measures, which is an additional limitation. We considered using frequency of rapid responses as a measure; however, these data would be highly biassed given that rapid responses are initiated by patients, families and providers for a variety of reasons, few of which are likely to relate to dysrhythmias. Overall hospital mortality would have been another measure to include; however, it is also affected by many factors that are not within the scope of this project.

Furthermore, during PDSA cycle 1, we did not have the personnel capacity to ensure that telemetry utilisation was asked about at every huddle. We relied on bedside nurses and nurse managers to carry this out, although it may have been done so inconsistently without our knowledge. During PDSA cycle 2, the intervention was designed to reduce inappropriate ordering of telemetry at the time of a patient's admission but it did not address starting telemetry after a patient was already admitted.

Finally, our measurement for telemetry utilisation was based on billing data that are linked to the ordered level of care for a patient on a given midnight. A provider may have stopped telemetry by writing a "nurse communication order' to remove the telemetry monitor, which would not have been reflected in the level of care billing data. As providers at this institution we think this is a rare event because our experience is that a nurse will clarify a 'nurse communication order' to discontinue telemetry by asking the provider to write a change in level of care order, as this is the expected practice pattern.

\section{CONCLUSION}

The overall goal of this project was to achieve a $20 \%$ reduction in hospital-wide telemetry utilisation and after our second PDSA cycle we approached this goal with a $17.8 \%$ relative decrease. We outlined guideline-based reasons for telemetry use in our PDSA cycle 2 intervention and thus we believe that inappropriate utilisation was the greatest area reduced. The intervention of introducing a hard-stop into admission orders is easily accomplished and easily measurable. Although our findings are from a single institution, other investigators have found similar results when investigating the use of prompts or 'hardwiring' order sets to minimise the use of telemetry not supported by the AHA guidelines. ${ }^{11} 20$

We observed differences in reductions of telemetry utilisation between different hospital units. For example, Unit B had a continued decrease in telemetry use after PDSA cycle 2, whereas Unit E did not. We hypothesise these differences are due to the patient populations and frequency of clinical problems on different hospital units. Some units may have a higher rate of inappropriate telemetry utilisation and thus would be more affected towards reduction with even a single intervention. Other units may have less inappropriate utilisation and reductions are not as great because the baseline opportunity for improvement is not as great. Furthermore, every unit will likely have a 'floor' level for utilisation, which would signify it has reached an appropriate level of telemetry utilisation and further reductions are not feasible or prudent.

Similar to other studies in this area, we achieved greatest reductions by incorporating hard-stops into the EMR. In a medium-sized study of 196 patients, Leighton et al showed reduction of initiation of telemetry with use of a hard-stop in the EMR. ${ }^{13}$ The notion holds true in our study that a preventive measure of keeping providers from inappropriately ordering telemetry monitoring in the first place has the greatest effect. Svec et al showed a reduction in telemetry utilisation at an academic medical centre by utilising a huddle strategy; however, they also provided a financial incentive to the individual provider for reducing his/her telemetry utilisation. ${ }^{4}$ Our huddle intervention provided no incentive and relied on the good intentions of providers, which ultimately did not provide a lasting reduction. We hypothesise that telemetry might be stopped at the appropriate time by either tying incentives to a huddle intervention or by making use 
of an automated protocol to discontinue telemetry based on clinical criteria and/or amount of time the patient has already been monitored without dysrhythmia detection.

\section{FUTURE DIRECTIONS}

Placing a hard-stop into the admission orders was most effective in reducing inappropriate telemetry at our institution and remains in place to date. Because this quality improvement project addressed initiation of telemetry monitoring on admission, for the next PDSA cycle, we will test another intervention to reduce telemetry monitoring after admission. For example, for a patient who had a legitimate reason for initiation but is now stabilised and remains hospitalised for other reasons. For our next steps, we intend to investigate a nurse-driven telemetry discontinuation protocol to stop telemetry that is no longer indicated. A nurse would initiate this process and the physician or advanced practice provider will have an opportunity to decide whether to stop telemetry based on an algorithmic approach.

Acknowledgements The authors would like to acknowledge the efforts of a large multidisciplinary team collaborating at KUMC that helped to execute portions of the quality improvement programme described here.

Contributors KS designed the study as principal investigator and executed the interventions. He also contributed to the collection and analysis of data, and drafted and revised the manuscript. MB contributed to the overall study design and implementation. He also collected and analysed data, and participated in drafting and revising the manuscript. CS and RM analysed data, developed the figures and tables, and assisted with drafting and revising the manuscript. CG advised on the data collection process, facilitated data analysis and participated in drafting and revising the manuscript. All authors have reviewed and approve of the final manuscript.

Funding This QI project was unfunded.

Competing interests None declared.

Patient consent for publication This project was approved by the local institutional review board and designated as quality improvement.

Provenance and peer review Not commissioned; externally peer reviewed.

Open access This is an open access article distributed in accordance with the Creative Commons Attribution Non Commercial (CC BY-NC 4.0) license, which permits others to distribute, remix, adapt, build upon this work non-commercially, and license their derivative works on different terms, provided the original work is properly cited, appropriate credit is given, any changes made indicated, and the use is non-commercial. See: http://creativecommons.org/licenses/by-nc/4.0/.

\section{REFERENCES}

1. Crawford MH, Bernstein SJ, Deedwania PC, et al. ACC/AHA guidelines for ambulatory electrocardiography. A report of the American College of Cardiology/American Heart Association Task
Force on practice guidelines (Committee to revise the guidelines for ambulatory electrocardiography). developed in collaboration with the North American Society for pacing and electrophysiology. J Am Coll Cardiol 1999;34:912-48.

2. Ivonye $\mathrm{C}$, Ohuabunwo $\mathrm{C}$, Henriques-Forsythe $\mathrm{M}$, et al. Evaluation of telemetry utilization, policy, and outcomes in an inner-city academic medical center. J Natl Med Assoc 2010;102:598-604.

3. Sandau KE, Funk M, Auerbach A, et al. Update to practice standards for electrocardiographic monitoring in hospital settings: a scientific statement from the American Heart Association. Circulation 2017;136:e273-344.

4. Svec D, Ahuja N, Evans $\mathrm{KH}$, et al. Hospitalist intervention for appropriate use of telemetry reduces length of stay and cost. $J$ Hosp Med 2015;10:627-32.

5. Lee J, Lamb P, Rand $E$, et al. Optimizing telemetry utilization in an academic medical center. J Clin Outcomes Manage 2008;15:435-40.

6. Benjamin EM, Klugman RA, Luckmann R, et al. Impact of cardiac telemetry on patient safety and cost. Am J Manag Care 2013;19:e225-32.

7. Najafi N, Auerbach A. Use and outcomes of telemetry monitoring on a medicine service. Arch Intern Med 2012;172:1349-50.

8. Curry JP, Hanson CW, Russell MW, et al. The use and effectiveness of electrocardiographic telemetry monitoring in a community hospital general care setting. Anesth Analg 2003;97:1483-7.

9. Feder S, Funk M. Over-monitoring and alarm fatigue: for whom do the bells Toll? Heart Lung 2013;42:395-6.

10. Srinivasa E, Mankoo J, Kerr C. An evidence-based approach to reducing cardiac telemetry alarm fatigue. Worldviews Evid Based Nurs 2017;14:265-73.

11. Dressler R, Dryer MM, Coletti C, et al. Altering overuse of cardiac telemetry in non-intensive care unit settings by hardwiring the use of American Heart Association guidelines. JAMA Intern Med 2014;174:1852-4.

12. Chen $S$, Palchaudhuri $S$, Johnson $A$, et al. Does this patient need telemetry? An analysis of telemetry ordering practices at an academic medical center. J Eval Clin Pract 2017;23:741-6.

13. Leighton $\mathrm{H}$, Kianfar $\mathrm{H}$, Serynek $\mathrm{S}$, et al. Effect of an electronic ordering system on adherence to the American College of Cardiology/American Heart Association guidelines for cardiac monitoring. Crit Pathw Cardiol 2013;12:6-8.

14. Bulger J, Nickel W, Messler J, et al. Choosing wisely in adult hospital medicine: five opportunities for improved healthcare value. $J$ Hosp Med 2013;8:486-92.

15. Armaignac DL, Saxena A, Rubens M, et al. Impact of telemedicine on mortality, length of stay, and cost among patients in progressive care units: experience from a large healthcare system. Crit Care Med 2018;46:728-35.

16. Drew BJ, Califf RM, Funk M, et al. Practice standards for electrocardiographic monitoring in hospital settings: an American Heart Association scientific statement from the councils on cardiovascular Nursing, clinical Cardiology, and cardiovascular disease in the young: endorsed by the International Society of computerized Electrocardiology and the American Association of Critical-Care nurses. Circulation 2004;110:2721-46.

17. Powers EM, Shiffman RN, Melnick ER, et al. Efficacy and unintended consequences of hard-stop alerts in electronic health record systems: a systematic review. J Am Med Inform Assoc 2018;25:1556-66.

18. Capewell S, Capewell A. An effectiveness hierarchy of preventive interventions: Neglected paradigm or self-evident truth? J Public Health 2017:1-9.

19. Gosbee L. Using human factors engineering to improve patient safety: problem solving on the front line. Oakbrook, IL: The Joint Commission, 2010.

20. Rizvi W, Munguti CM, Mehta J, et al. Reducing Over-Utilization of cardiac telemetry with Pop-Ups in an electronic medical record system. Cureus 2017;9:e1282. 